\title{
Predictors for functional decline after an injurious fall: a population-based cohort study
}

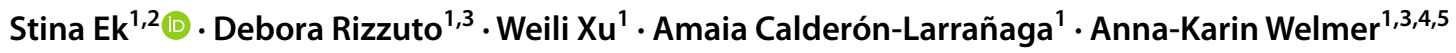

Received: 28 July 2020 / Accepted: 22 October 2020 / Published online: 7 November 2020

(c) The Author(s) 2020

\begin{abstract}
Background The functional consequences of injurious falls are well known. However, studies of the factors that can modify trajectories of disability after an injury from a fall are scarce.

Aims We aimed to investigate whether sociodemographic and health-related factors may impact this association.

Methods The study population consisted of 1426 community-dwelling older adults ( $\geq 60$ years) from the SNAC-K cohort study in Stockholm, Sweden. Functional status over 12 years of follow-up was assessed using the number of limitations in basic and instrumental activities of daily living. Sex, cohabitation status, physical activity, and self-rated health were assessed at baseline. Injurious falls were defined as falls requiring healthcare and were assessed over 3 years starting at baseline. Data were analyzed using linear-mixed effects models.

Results The fastest increase in the number of disabilities was observed in those who had endured an injurious fall and were living alone $(\beta$ coefficient $=0.408 ; p<0.001)$, been physically inactive $(\beta$ coefficient $=0.587 ; p<0.001)$, and had poor selfrated health $(\beta$ coefficient $=0.514 ; p<0.001)$. The negative impact of these factors was more pronounced among fallers compared to non-fallers.

Discussion Living alone, being physically inactive, and having poor self-rated health magnifies the negative effect of an injurious fall on functional status. Among individuals who endure an injurious fall, the heterogeneity in long-term functional status is substantial, depending on the individuals' characteristics and behaviors.

Conclusions These findings emphasize the need for a person-centered approach in care provision and can guide secondary prevention within health care.
\end{abstract}

Keywords Older adults $\cdot$ IADL $\cdot$ ADL $\cdot$ Disability trajectories $\cdot$ SNAC-K

\section{Introduction}

Injurious falls are the most common cause of hospitalization among older adults [1]. The consequences of such falls may be detrimental in terms of functional outcomes, leading

Electronic supplementary material The online version of this article (https://doi.org/10.1007/s40520-020-01747-1) contains supplementary material, which is available to authorized users.

Stina Ek

stina.ek@ki.se

1 Aging Research Center (ARC), Department of Neurobiology, Care Sciences and Society, Karolinska Institutet and Stockholm University, Stockholm, Sweden

2 Unit of Epidemiology, the Institute of Environmental Medicine, Karolinska Institutet, Box 210, 17177 Stockholm, Sweden to decreased quality of life for the individual and a high care burden for society [2]. Functional decline is most often defined by increased dependency in basic and instrumental activities of daily living [3-5]. Indeed, maintaining independence while aging is among the most important dimensions of health for older individuals themselves, and should, therefore, be prioritized [6]. A proper understanding of the long-term functional consequences of an injurious fall can

3 Stockholm Gerontology Research Center, Stockholm, Sweden

4 Allied Health Professionals, Function Area Occupational Therapy \& Physiotherapy, Karolinska University Hospital, Stockholm, Sweden

5 Division of Physiotherapy, Department of Neurobiology, Care Sciences and Society, Karolinska Institutet, Stockholm, Sweden 
guide care planning, rehabilitation, and resource allocation. While the functional consequences of an injurious fall are well established, possible factors that could impact this association are not well-understood. A few studies have shown that certain traits and characteristics can impact the trajectory of physical decline and dependence among older adults that have endured a fall [3, 7], but more studies are needed to establish which specific health-related and sociodemographic factors determine the disability trajectories of older adults that have endured an injurious fall.

Previous studies, including our research, suggest that men and women may differ in terms of risk factors for falls [8-10]. However, only a few studies have investigated whether recovery of functional status differs between men and women who survive a fall-injury hospitalization, and with inconsistent results [7, 11-13]. Living with someone has been shown to improve the course of disability after an injury [7], and findings from our research group have indicated that living alone is an important risk factor for injurious falls [14]. Both low physical activity and poor self-rated health are known risk factors for falls [15], and inactivity and poor health-related quality of life have shown to be consequences of an injury $[2,16]$.

In conclusion, there is a need to identify both modifiable and non-modifiable factors that may impact the development of functional decline after an injurious fall to determine which individuals are most at risk of not reaching full recovery. In this population-based study of older adults, we aimed to investigate the sociodemographic and health-related predictors of disability trajectories after an injurious fall.

\section{Methods}

\section{Study population}

We gathered data from the Swedish National Study on Aging and Care in Kungsholmen (SNAC-K) [17]. The population of Kungsholmen in central Stockholm, Sweden, was randomly sampled from 11 age cohorts $(60,66,72,78,81,84$, 87, 90, 93, 96 and 99+). In total, 4590 people were invited and 3363 participated (response rate $73.3 \%$ ) in the baseline assessment (2001-2004). SNAC-K is an ongoing longitudinal study; people aged 60-72 are assessed every sixth year while people aged over 78 are assessed every third year. Participants in this study were followed for up to 12 years (2013-2016).

Of the total sample, we excluded participants who refused the use of their health register data $(n=62)$. Due to differences in post-acute care and to establish the exposure period, we also excluded those people who lived in nursing homes $(n=189)$ and those who had fallen after 3 years since baseline $(n=291)$. Furthermore, we excluded people who did not participate in any SNAC-K follow-up assessments $(n=1050)$ and those with missing data for any of the covariates or exposures $(n=251)$. The analytical sample consisted of 1426 individuals. The excluded participants were older, had lower levels of education, had a greater number of disabilities, were less physically active, and were more likely to be women than those in the analytical sample $(p<0.05)$.

SNAC-K was approved by the Regional Ethical Board in Stockholm and follows the Helsinki Declaration. All participants provided written informed consent and are free to leave the study whenever they wish to.

\section{Data assessment}

In SNAC-K, trained nurses and physicians collected data based on structured interviews, clinical examinations and physical function tests. The full SNAC-K protocol is available at https://www.snac.org.

\section{Outcome}

Independence in activities of daily living (ADL) and instrumental ADL (IADL) were assessed through interviews. ADL included bathing, getting dressed, toileting, transferring, and eating. IADL included managing finances, using the telephone, grocery shopping, using public transportation, preparing meals, doing laundry, and cleaning. ADL and IADL items were combined into a disability score with a value ranging from 0 to 14 [18].

\section{Exposures}

An injurious fall was defined as a fall requiring inpatient or outpatient specialist care. The following discharge diagnoses from the International Classification of Diseases 10th revision (ICD-10) were used: W00, W01, W05-W10, and W17-W19. These were chosen to represent a fall caused by low force with no other person involved. The Swedish personal identification number (PIN) was used to link data from the National Patient Register and the Local Outpatient Register to each SNAC-K participant [19, 20]. In this study, we included injurious falls that occurred up to 3 years after the baseline examination as an exposure. Sex was derived from registers. Cohabitation status was assessed through interviews; people who were married or cohabiting were classified as living with someone, whereas unmarried, divorced, and widowed people were classified as living alone. Physical activity level was determined based on two questions from a self-administered questionnaire: (1) "Do you regularly engage in light exercise? (such as walking, shorter bicycling, and golf)" and (2) "Do you regularly engage in more intense exercise? (such as jogging or brisk long walks, heavy-duty gardening, high-intensity aerobics, skiing, swimming, and 
ball sports)." Both questions concerned the last 12 months and possible answers included: every day, several times per week, 2-3 times per month, less, and never. The two questions were combined in one dichotomous variable (inactive/ active), based on current guidelines. Participants were considered active if they were engaged in light and/or intense exercise every day or several times per week and inactive for any of the other response options [21]. Self-rated health was assessed by a questionnaire including the question "In general, how would you describe your health?"; the answers "poor" and "fair" were categorized as "poor" while the answers "good", "very good," and "excellent" were categorized as "good".

\section{Covariates}

Age was derived from registers. Education was assessed through interviews and refers to the highest level of education achieved (elementary, high school, or university). Cognition was assessed with the Mini-Mental State Examination (MMSE) test and cognitive impairment was defined as having a MMSE score of less than 28, because of the highly educated population studied [22]. Multimorbidity was defined as having two or more chronic diseases from a list developed by an interdisciplinairy team in a previous study by Calderón-Larrañaga et al. [23]. A disease was defined as chronic if it had a prolonged duration and either (1) led to residual disability or worsening quality of life, or (2) required long period of care, treatment, or rehabilitation. In SNAC-K, chronic conditions were determined based on a combination of clinical examination data, laboratory data, current drug use and health care register data. Data on survival was extracted from the Swedish Death Registers and was linked to the participants by the PIN. Survival status was measured throughout the whole follow-up and was categorized as alive or dead at the end of the study period.

\section{Statistical analysis}

Each binary predictor-sex, cohabitation status, physical activity level, and self-rated health-was combined with injurious falls (falls/no falls) to create four different indicator variables, each with four mutually exclusive categories. Linear mixed-effects models with random effects for intercept and slope were used to examine the association between the indicator variables and the changes in disability score over time, resulting in four separate models. To measure the effect of the exposures on the average annual change in the number of disabilities, the interaction term between followup time (in years) and each of the four indicator variables was included as a fixed effect. All four models were adjusted for all other exposures, as well as age, education level, multimorbidity, and MMSE score. Survival was also included in the models initially but was omitted due to collinearity with other covariates. Non-linearity of follow-up time was tested but was not significant. Interactions between injurious falls and sex, living alone, inactivity, and self-rated health on disability were also tested separately.

\section{Sensitivity analysis}

To verify that the results were not driven by injurious falls that occurred before the baseline examination or by the severity of the injury (i.e. experiencing a fracture), we performed the following sensitivity analyses: (1) excluding individuals who had experienced an injurious fall within 3 years of the baseline assessment, and (2) defining injurious falls as falls that resulted in fractures. Finally, to take into accunt the missing data, multivariate imputation by chained equations (MICE) [24] was performed to obtain five imputed datasets. All variables included in the main analyses were used in the multiple imputation models.

\section{Results}

Of the 1426 individuals in the study sample, 867 (60.8\%) were women and the mean age was 69.3 (SD 8.5). Seventynine individuals (5.5\%) experienced a fall between baseline and the three-year follow-up examination. Baseline characteristics of the analytical sample are presented in Table 1.

Data on baseline health status and the number of deaths and dropouts in the different study groups over the 12-year follow-up are presented in Table 2.

Results from the linear mixed effects models indicated that women had more disabilities than men at baseline

Table 1 Baseline characteristics of the analytical sample, $n=1426$

\begin{tabular}{ll}
\hline Characteristics & Sample, $N=1426$ \\
\hline Age, mean \pm SD & $69.3 \pm 8.5$ \\
Women, $n(\%)$ & $867(60.8)$ \\
Education, $n(\%)$ & \\
Elementary & $148(10.4)$ \\
High School & $681(47.8)$ \\
University & $597(41.9)$ \\
Multimorbidity, $n(\%)$ & $1157(81.1)$ \\
MMSE <28, $n(\%)$ & $94(6.6)$ \\
Living alone, $n(\%)$ & $643(45.1)$ \\
Physically inactive, $n(\%)$ & $255(17.9)$ \\
Fair to poor self-rated health, $n(\%)$ & $302(21.2)$ \\
Fallers between baseline and 3 years, $n(\%)$ & $79(5.5)$ \\
Previous fallers (3 years prior to baseline), $n(\%)$ & $66(4.6)$ \\
Number of disabilities at baseline, mean \pm SD & $0.1 \pm 0.6$ \\
\hline
\end{tabular}

Multimorbidity $=2$ or more diseases, $M M S E$ mini mental state examination, physically inactive $\leq 1$ activity/week 
Table 2 Distribution of baseline multimorbidity, cognitive impairment, previous falls and number of deaths and dropouts during the 12-year follow-up, by the different groups of combinations of injurious falls with sex, cohabitation status, physical activity level and self-rated health

\begin{tabular}{|c|c|c|c|c|c|c|}
\hline & $n$ & $\begin{array}{l}\text { Baseline multi- } \\
\text { morbidity (\%) }\end{array}$ & $\begin{array}{l}\text { Baseline cognitive } \\
\text { impairment }(\%)\end{array}$ & Previous falls (\%) & $\begin{array}{l}\text { Number of deaths at } \\
12 \text {-year follow-up (\%) }\end{array}$ & $\begin{array}{l}\text { Number of dropouts at } \\
12 \text {-year follow-up (\%) }\end{array}$ \\
\hline \multicolumn{7}{|l|}{ Sex } \\
\hline Man, no fall & 541 & 425 (78.6) & $40(7.4)$ & $12(2.2)$ & $111(20.5)$ & $61(11.3)$ \\
\hline Woman, no fall & 806 & $660(81.9)$ & $44(5.5)$ & $41(5.1)$ & $127(15.8)$ & $114(14.2)$ \\
\hline Man, fall & 18 & $16(88.9)$ & $2(11.1)$ & $3(16.7)$ & $9(50.0)$ & $1(5.6)$ \\
\hline Woman, fall & 61 & $56(91.8)$ & $8(13.1)$ & $10(16.4)$ & $19(31.2)$ & $8(13.1)$ \\
\hline \multicolumn{7}{|l|}{ Cohabitation } \\
\hline Cohabiting, no fall & 759 & $589(77.6)$ & $36(4.7)$ & $24(3.2)$ & $104(13.7)$ & $95(12.5)$ \\
\hline Alone, no fall & 588 & $496(84.4)$ & $48(8.2)$ & $29(4.9)$ & $134(22.8)$ & $80(13.6)$ \\
\hline Cohabiting, fall & 24 & $22(91.7)$ & $1(4.2)$ & $2(8.3)$ & $7(29.2)$ & $2(8.3)$ \\
\hline Alone, fall & 55 & $50(90.9)$ & $9(16.4)$ & $11(20.0)$ & $21(38.2)$ & $7(12.7)$ \\
\hline \multicolumn{7}{|l|}{ Physical activity } \\
\hline Active, no fall & 1113 & $895(80.4)$ & $66(5.9)$ & $41(3.7)$ & $192(17.3)$ & $136(12.2)$ \\
\hline Inactive, no fall & 234 & $190(81.2)$ & $18(7.7)$ & $12(3.1)$ & $46(19.8)$ & $39(16.8)$ \\
\hline Active, fall & 58 & $51(87.9)$ & $7(12.1)$ & $7(12.1)$ & $16(27.6)$ & $7(12.1)$ \\
\hline Inactive, fall & 21 & $21(100.0)$ & $3(14.3)$ & $6(28.6)$ & $12(57.1)$ & $2(9.5)$ \\
\hline \multicolumn{7}{|l|}{ Self-rated health } \\
\hline Good, no fall & 1083 & $832(76.8)$ & $61(5.6)$ & $40(3.7)$ & $153(14.1)$ & $141(13.0)$ \\
\hline Poor, no fall & 264 & $253(95.8)$ & $23(8.7)$ & $13(4.9)$ & $85(32.3)$ & 34 (12.9) \\
\hline Good, fall & 41 & $36(87.8)$ & $7(17.1)$ & $7(17.1)$ & $9(22.0)$ & $5(12.2)$ \\
\hline Poor, fall & 38 & $36(94.7)$ & $3(7.9)$ & $6(15.8)$ & $19(50.0)$ & $4(10.5)$ \\
\hline
\end{tabular}

regardless of the future occurrence of a fall, although there were no sex differences in the disability trajectory over time (Table 3; Fig. 1). In terms of falls and cohabitation status, there were no differences in disability levels at baseline, but the number of disabilities increased fastest for fallers who lived alone $(\beta$ coefficient $=0.408 ; p<0.001)$. Physically inactive individuals had more disabilities than active individuals at baseline for both fallers and non-fallers, but the steepest disability trajectory was seen for inactive fallers $(\beta$ coefficient $=0.587 ; p<0.001)$. Individuals with poor self-rated health had higher levels of disability at baseline compared to those with good self-rated health, regardless of future falls, but the highest increase in disability was seen for fallers with poor self-rated health $(\beta$ coefficient $=0.514$; $p<0.001)$. In addition, non-fallers with poor self-rated health had an almost identical increase in disability as fallers with good self-rated health. The increase in the number of disabilities was higher among non-cohabiting vs. cohabiting fallers compared to non-cohabiting vs. cohabiting nonfallers ( $\beta$ coefficients 0.205 and 0.059 , respectively), inactive vs. active fallers compared to inactive vs. active non-fallers ( $\beta$ coefficients 0.337 and 0.112 , respectively), and fallers with poor vs. good self-rated health compared to non-fallers with poor vs. good self-rate health ( $\beta$ coefficients: 0.394 and 0.153 , respectively) (Table 3; Fig. 1). The interaction between sex and falls on disability was not significant, but the interactions between falls and the other three exposures (living alone, inactivity and self-rated health) were $(p<0.05)$.

\section{Sensitivity analyses}

The analyses excluding previous fallers and when only considering injurious falls leading to fractures yielded similar results to the original analyses, suggesting that neither injurious falls that occurred before the baseline examination nor the severity of the injury (i.e. experiencing a fracture) was driving the results (Supplementary Tables 1 and 2). Finally, results from the imputed datasets were similar to those from the complete case analyses (Supplementary Table 3).

\section{Discussion}

In this population-based longitudinal study of older adults, we found that living alone, being physically inactive, and having a poor self-rated health predicted steeper declines in disability over a 12-year period, and these differences were even greater among fallers compared to non-fallers.

The predictors explored in this study can be categorized into modifiable and non-modifiable factors. Modifiable factors, such as physical activity, can be targeted for either 
Table $3 \beta$ coefficient and 95\% confidence intervals (CI) for the association between injurious falls in combination with sex, cohabitation, physical activity level and self-rated health and changes in disability over 12 years

\begin{tabular}{|c|c|c|c|c|c|c|c|}
\hline & $n$ & Baseline, $\beta$ & $(95 \% \mathrm{CI})$ & $p$ & Annual change, $\beta$ & $95 \% \mathrm{CI}$ & $p$ \\
\hline \multicolumn{8}{|l|}{ Sex } \\
\hline Man, no fall & 541 & Ref & & & Ref & & \\
\hline Woman, no fall & 806 & 0.105 & $0.000-0.209$ & 0.048 & 0.014 & -0.017 to 0.047 & 0.365 \\
\hline Man, fall & 18 & 0.109 & -0.318 to 0.536 & 0.616 & 0.341 & $0.200-0.482$ & $<0.001$ \\
\hline Woman, fall & 61 & 0.310 & $0.066-0.554$ & 0.013 & 0.324 & $0.246-0.402$ & $<0.001$ \\
\hline \multicolumn{8}{|l|}{ Cohabitation } \\
\hline Cohabiting, no fall & 759 & Ref & & & Ref & & \\
\hline Alone, no fall & 588 & -0.068 & -0.172 to 0.037 & 0.203 & 0.059 & $0.027-0.090$ & $<0.001$ \\
\hline Cohabiting, fall & 24 & -0.062 & -0.434 to 0.310 & 0.742 & 0.203 & $0.086-0.320$ & $<0.001$ \\
\hline Alone, fall & 55 & 0.229 & -0.023 to 0.480 & 0.075 & 0.408 & $0.328-0.489$ & $<0.001$ \\
\hline \multicolumn{8}{|l|}{ Physical activity } \\
\hline Active, no fall & 1113 & Ref & & & Ref & & \\
\hline Inactive, no fall & 234 & 0.187 & $0.058-0.316$ & 0.005 & 0.112 & $0.071-0.152$ & $<0.001$ \\
\hline Active, fall & 58 & -0.082 & -0.319 to 0.154 & 0.495 & 0.250 & $0.174-0.326$ & $<0.001$ \\
\hline Inactive, fall & 21 & 1.123 & $0.731-1.517$ & $<0.001$ & 0.587 & $0.458-0.717$ & $<0.001$ \\
\hline \multicolumn{8}{|l|}{ Self-rated health } \\
\hline Good, no fall & 1083 & Ref & & & Ref & & \\
\hline Poor, no fall & 264 & 0.194 & $0.067-0.321$ & 0.003 & 0.153 & $0.115-0.192$ & $<0.001$ \\
\hline Good, fall & 41 & -0.034 & -0.314 to 0.245 & 0.811 & 0.120 & $0.112-0.288$ & $<0.001$ \\
\hline Poor, fall & 38 & 0.663 & $0.358-0.959$ & $<0.001$ & 0.514 & $0.420-0.609$ & $<0.001$ \\
\hline
\end{tabular}

Controlled for age, education, multimorbidity, MMSE and the other exposure variables (sex, living alone, physical activity level and selfreported health) when applicable. Significant $p$ values on a 95\% confidence interval level in bold

primary prevention (i.e., prevention of falls) as well tertiary prevention (i.e., prevention of the long-lasting complications due to falls), by encouraging increased physical activity levels in the general older population [25]. In addition, the number of disabilities at baseline already differed between future fallers and non-fallers, by physical activity level and self-rated health. This could indicate that the injurious fall is a consequence rather than a cause of the increased disability level. Findings from Gill et al. might support this: They demonstrated that the course of disability before a fall is highly interrelated with the course of disability after a fall [26].

In this study, we did not find an association between sex and disability trajectories after an injurious fall. Previous studies on this have shown inconsistent results: one study concluded that women have a better prognosis [12], another study reported that men regain higher levels of physical function [11], and yet another showed no significant differences between men and women [13]. In this study, we controlled for health-related factors that are known to differ between men and women (e.g., age, education, cognition and multimorbidity), which may explain the non-significant result reported here. In addition, as shown in Table 2, men had a lower rate of survival in this study, which may result in a selection bias for the men.
In line with Bell et al.'s results, we showed that individuals living with a spouse had a better functional trajectory after an injury [7]. Social support has shown to be important for successful recovery after an injury [27], and the mechanisms behind this could relate not only to physical and emotional support but also adherence to treatment. DiMatteo et al. have shown in a meta-analysis that adherence to treatment is associated with social support [28].

Our results showed that being physically inactive prior to an injurious fall predicted a worse disability trajectory. Physical activity interventions with the aim to both reduce the risk of falls and to enhance the activity level after a fall have shown positive results $[29,30]$, confirming that such interventions might be an optimal primary and also tertiary prevention strategy to decrease the burden of falls among older adults.

Our results indicate that self-rated health prior to a fall is strongly associated with the course of disability, even after controlling for objective health-related factors such as age, multimorbidity and cognitive status. This is in line with the findings of Brenowitz et al. who showed that low self-rated health predicted decline in physical function [31]. Self-rated health is a comprehensive concept that may reflect not only an individual's spontaneous view of their current health but also their health-related goals [32]. It appears 

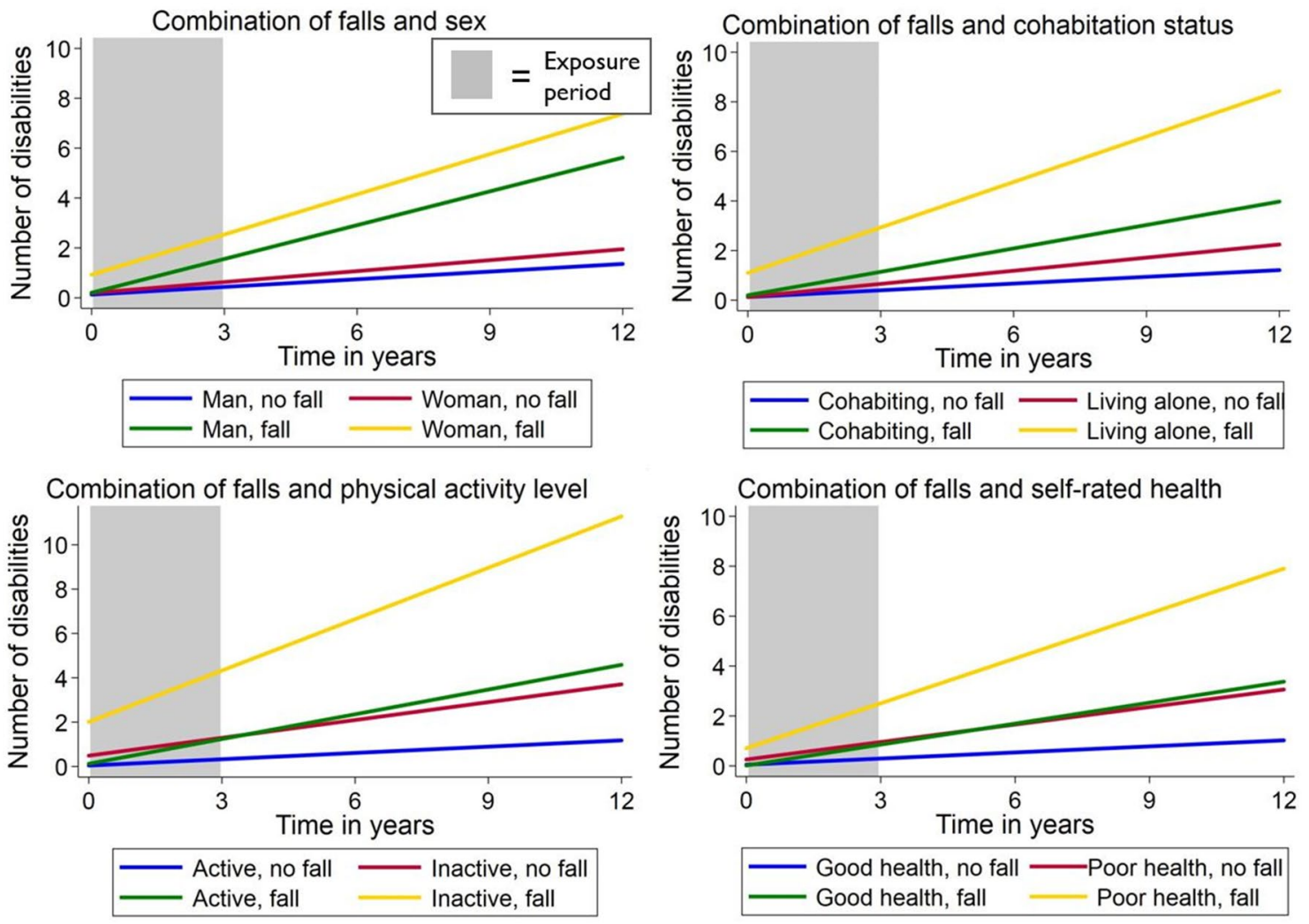

Fig. 1 Predicted mean number of disabilities associated with injurious falls in combination with sex, cohabitation, physical activity level and self-rated health

that an individual's subjective perception of health can alter the functional outcome after an adverse event, regardless of their objective health. This might reflect an individual's resilience, including their health perceptions and expectations. Indeed, Ayalon et al. showed that satisfaction with aging can be protective against falls and suggested psychosocial interventions to decrease the risk of falling [33].

As for practical applications, the results from this study can be viewed as a basis for detecting individuals who are especially vulnerable to a worse prognosis after an injurious fall, with a possible underlying frailty phenotype [34]. Our findings also provide guidance regarding the maintenance of function and independence for individuals who fall through tertiary prevention. This is in line with the concept of physical resilience, which emphasizes the need to enhance and focus on protective factors instead of risk factors [35].

The proportion of fallers that died or dropped out from the study before the end of the follow-up was significantly higher than that of non-fallers, indicating that primary prevention measures to decrease falls among older adults is an essential tool to increase not only life-expectancy but also disability-free life years [36].

Strengths of this study include the use of population-based data including a number of objective health measurements as well as the linkage of this data to an almost full-coverage health care register, minimizing the risk of recall bias. Furthermore, the long follow-up period in this study adds novel information to research in this field. However, it is possible that factors impacting functional decline changed between waves of data collection in SNAC-K, and this was not considered in our study. There are also other limitations to consider. Some of the variables used in this study - although validated and commonly used in clinicare rather crude measures lacking in detail; this includes the MMSE, the aggregate measure of ADL/IADL, and the definition of multimorbidity as $\geq 2$ diseases. Additionally, mortality can be a competing risk when following older individuals for such a long time, especially among the subgroup of fallers. That said, survival status was omitted from the models due to collinearity, and death did not affect the disability trajectories beyond the other covariates. Finally, the study population comes from a wealthy area and may not be representative of the general aging population. Thus, our results need to be confirmed in other cohorts.

In conclusion, this study suggests that it is possible to predict functional decline in older adults after a fall using sociodemographic and health-related factors. After controlling for potential confounders, we found that living alone, 
being physically inactive, and having a poor self-rated health predicted a steeper decline in disability, and these differences were even more accentuated among fallers compared to non-fallers. This indicates that even among individuals who endured an injurious fall there is substantial heterogeneity in long-term functional status depending on individual characteristics and behaviors, emphasizing the need for a person-centered approach in care provision. In addition, some of these characteristics, such as physical activity level, are modifiable and can be targeted for primary as well as tertiary prevention of falls.

Acknowledgements This study was accomplished within the context of the Swedish National Graduate School for Competitive Science on Ageing and Health (SWEAH) funded by the Swedish Research Council. The authors would also like to pay their gratitude to the data collection staff for their invaluable contributions.

Author contributions SE: conceptualization, data curation, formal analysis, methodology, writing-original draft. DR: methodology, supervision, writing - review and editing. WX: supervision, writing-review and editing. ACL: conceptualization, writing-review and editing. AKW: data curation, funding acquisition, methodology, supervision, writing - review and editing.

Funding Open access funding provided by Karolinska Institute. The Swedish National Study on Aging and Care in Kungsholmen is supported by the Swedish Ministry of Health and Social Affairs and the participating county councils and municipalities. This study was further supported by the Swedish Research Council (Grant Number 521-201421-96) and Gun and Bertils Stohnes Stiftelse.

\section{Compliance with ethical standards}

\section{Conflicts of interest None.}

Ethics approval and Informed consent SNAC-K is approved by the Regional Ethical Board in Stockholm and follows the Helsinki Declaration. All participants have given informed written consent and can drop out of the study whenever they wish to.

Open Access This article is licensed under a Creative Commons Attribution 4.0 International License, which permits use, sharing, adaptation, distribution and reproduction in any medium or format, as long as you give appropriate credit to the original author(s) and the source, provide a link to the Creative Commons licence, and indicate if changes were made. The images or other third party material in this article are included in the article's Creative Commons licence, unless indicated otherwise in a credit line to the material. If material is not included in the article's Creative Commons licence and your intended use is not permitted by statutory regulation or exceeds the permitted use, you will need to obtain permission directly from the copyright holder. To view a copy of this licence, visit http://creativecommons.org/licenses/by/4.0/.

\section{References}

1. World Health Organization (2007) WHO global report on falls prevention in older age. World Health Organization (WHO), Geneva
2. Peeters GM, Jones M, Byles J et al (2015) Long-term consequences of noninjurious and injurious falls on well-being in older women. J Gerontol A Biol Sci Med Sci. 70:1519-1525

3. Stel VS, Smit JH, Pluijm SMF et al (2004) Consequences of falling in older men and women and risk factors for health service use and functional decline. Age Ageing 33:58-65

4. Gill TM, Murphy TE, Gahbauer EA et al (2013a) Association of injurious falls with disability outcomes and nursing home admissions in community-living older persons. Am J Epidemiol 178:418-425

5. Crandall CJ, LaMonte MJ, Snively BM et al (2016) Physical functioning among women aged 80 years and older with previous fracture. J Gerontol A Biol Sci Med Sci 71:S31-41

6. World Health Organization (2015) World report on ageing and health. World Health Organization (WHO), Geneva

7. Bell TM, Wang J, Nolly R et al (2015) Predictors of functional limitation trajectories after injury in a nationally representative U.S. older adult population. Ann Epidemiol 25:894-900

8. Ek S, Rizzuto D, Fratiglioni L et al (2019) Risk factors for injurious falls in older adults: the role of sex and length of follow-up. J Am Geriatr Soc 67:246-253

9. Chang VC, Do MT (2015) Risk factors for falls among seniors: implications of gender. Am J epidemiol 181:521-531

10. Gale CR, Westbury LD, Cooper C et al (2018) Risk factors for incident falls in older men and women: the English longitudinal study of ageing. BMC Geriatr 18:117

11. Arinzon Z, Shabat S, Peisakh A et al (2010) Gender differences influence the outcome of geriatric rehabilitation following hip fracture. Arch Gerontol Geriatr 50:86-91

12. Di Monaco M, Castiglioni C, Vallero F et al (2012) Men recover ability to function less than women do: an observational study of 1094 subjects after hip fracture. Am J Phys Med Rehabil 91:309-315

13. Beaupre LA, Carson JL, Noveck H et al (2015) Recovery of walking ability and return to community living within 60 days of hip fracture does not differ between male and female survivors. J Am Geriatr Soc 63:1640-1644

14. Ek S, Rizzuto D, Calderon-Larranaga A et al (2019) Predicting first-time injurious falls in older men and women living in the community: development of the first injurious fall screening tool. J Am Med Dir Assoc. 20:1163-1168.e3

15. Deandrea $S$, Lucenteforte E, Bravi F et al (2010) Risk factors for falls in community-dwelling older people: a systematic review and meta-analysis. Epidemiology 21:658-668

16. Stenhagen M, Ekstrom H, Nordell E et al (2014) Accidental falls, health-related quality of life and life satisfaction: a prospective study of the general elderly population. Arch Gerontol Geriatr 58:95-100

17. Lagergren M, Fratiglioni L, Hallberg IR et al (2004) A longitudinal study integrating population, care and social services data. The Swedish National study on Aging and Care (SNAC). Aging Clin Exp Res 16:158-168

18. LaPlante MP (2010) The classic measure of disability in activities of daily living is biased by age but an expanded IADL/ADL measure is not. J Gerontol B Psychol Sci Soc Sci 65:720-732

19. Ludvigsson JF, Otterblad-Olausson P, Pettersson BU et al (2009) The Swedish personal identity number: possibilities and pitfalls in healthcare and medical research. Eur J Epidemiol 24:659-667

20. Bergstrom MF, Byberg L, Melhus H et al (2011) Extent and consequences of misclassified injury diagnoses in a national hospital discharge registry. Inj Prev 17:108-113

21. Dohrn I, Gardiner PA, Winkler E et al (2020) Device-measured sedentary behavior and physical activity in older adults differ by demographic and health-related factors. Eur Rev Aging Phys Act 11:8 
22. O'Bryant SE, Humphreys JD, Smith GE et al (2008) Detecting dementia with the mini-mental state examination in highly educated individuals. Arch Neurol 65:963-967

23. Calderon-Larranaga A, Vetrano DL, Onder G et al (2016) Assessing and measuring chronic multimorbidity in the older population a proposal for its operationalization. J Gerontol A Biol Sci Med Sci. https://doi.org/10.1093/gerona/glw233

24. White IR, Royston P, Wood AM (2011) Multiple imputation using chained equations: issues and guidance for practice. Stat Med 30:377-399

25. Ania Z, Stephen M, Helen F et al (2017) Promotion of physical activity interventions for community dwelling older adults: A systematic review of reviews. PLoS ONE 12:e0180902

26. Gill TM, Murphy TE, Gahbauer EA et al (2013b) The course of disability before and after a serious fall injury. JAMA Intern Med 173:1780-1786

27. Kiely JM, Brasel KJ, Weidner KL et al (2006) Predicting quality of life six months after traumatic injury. J Trauma 61:791-798

28. DiMatteo MR (2004) Social support and patient adherence to medical treatment: a meta-analysis. Health Psychol 23:207-218

29. Gregg EW, Pereira MA, Caspersen CJ (2000) Physical activity, falls, and fractures among older adults: a review of the epidemiologic evidence. J Am Geriatr Soc 48:883-893

30. Sherrington C, Tiedemann A, Cameron I (2011) Physical exercise after hip fracture: an evidence overview. Eur J Phys Rehabil Med 47:297-307
31. Brenowitz WD, Hubbard RA, Crane PK et al (2014) Longitudinal associations between self-rated health and performance-based physical function in a population-based cohort of older adults. PLoS ONE 9:e111761

32. Bailis DS, Segall A, Chipperfield JG (2003) Two views of selfrated general health status. Soc Sci Med. 56:203-217

33. Ayalon L (2016) Satisfaction with aging results in reduced risk for falling. Int Psychogeriatr 28:741-747

34. Fried LP, Tangen CM, Walston J et al (2001) Frailty in older adults: evidence for a phenotype. J Gerontol A Biol Sci Med Sci 56:M146-M156

35. Whitson HE, Duan-Porter W, Schmader KE et al (2016) Physical resilience in older adults: systematic review and development of an emerging construct. J Gerontol A Biol Sci Med Sci 71:489-495

36. Prince MJ, Wu F, Guo Y et al (2015) The burden of disease in older people and implications for health policy and practice. Lancet 385:549-562

Publisher's Note Springer Nature remains neutral with regard to jurisdictional claims in published maps and institutional affiliations. 